\title{
On a question regarding visibility of lattice points, II
}

\author{
by \\ Sukumar Das AdHikari (Allahabad) and \\ Yong-GAo Chen (Nanjing)
}

1. Introduction. For $d \geq 2$, let $\Delta_{n}^{d}=\left\{\left(x_{1}, \ldots, x_{d}\right): x_{i}\right.$ integers and $\left.1 \leq x_{i} \leq n \forall i\right\}$ be the set of integer lattice points in a cube in $\mathbb{R}^{d}$. If $\alpha=\left(a_{1}, \ldots, a_{d}\right)$ and $\beta=\left(b_{1}, \ldots, b_{d}\right)$ are two points in $\Delta_{n}^{d}$, we say that $\alpha$ is visible from $\beta$ if either $\alpha=\beta$ or there is no lattice point in $\Delta_{n}^{d}$ on the line segment joining $\alpha$ and $\beta$. It is not difficult to verify that if $\alpha \neq \beta$, then $\alpha=\left(a_{1}, \ldots, a_{d}\right)$ is visible from $\beta=\left(b_{1}, \ldots, b_{d}\right)$ if and only if $\operatorname{gcd}\left(a_{1}-b_{1}, \ldots, a_{d}-b_{d}\right)=1$.

If $A$ and $B$ are subsets of $\Delta_{n}^{d}$, one says that $A$ is visible from $B$ if each point of $A$ is visible from some point of $B$.

Let $f_{d}(n)$ be defined by

$$
f_{d}(n)=\min \left\{|S|: S \subset \Delta_{n}^{d}, \Delta_{n}^{d} \text { is visible from } S\right\} .
$$

That is, $f_{d}(n)$ is the least number of points that can be selected from $\Delta_{n}^{d}$ such that every point of $\Delta_{n}^{d}$ is visible from at least one of the selected points.

Professor Imre Z. Ruzsa informed the authors that the problem of finding the exact order of $f_{d}(n)$ is one of the problems in the list compiled by L. \& W. Moser.

For the two-dimensional case, it was proved in [1] that

TheOREM $1^{*}$. For all $n>n_{0}$,

$$
\frac{\log n}{2 \log \log n}<f_{2}(n)<4 \log n
$$

Here, the second inequality was established by the greedy algorithm, while the first one follows by using the Chinese Remainder Theorem.

The following result was also proved in [1].

1991 Mathematics Subject Classification: Primary 11H99; Secondary 11B75. 
Theorem $2^{*}$. One can explicitly describe a set $S_{n} \subset \Delta_{n}^{2}$ such that $\Delta_{n}^{2}$ is visible from $S_{n}$ and $\left|S_{n}\right|=O\left((\log n)^{\alpha}\right)$ where $\alpha$ has the property that the Jacobsthal function $g(n)$ satisfies $g(n)=O\left((\log n)^{\alpha}\right)$.

Here, the Jacobsthal function $g(n)$ is defined to be the least integer with the property that among any $g(n)$ consecutive integers $a+1, \ldots$, $a+g(n)$, there is at least one which is relatively prime to $n$. Erdös [3] was the first to establish that $g(n)=O\left((\log n)^{\alpha}\right)$ for some finite $\alpha$. Since then, several mathematicians (see, for example, [5]-[9]) have taken up the problem of improving the estimate of Erdős. Currently, the best known result in this direction, due to Iwaniec [6], implies $g(n)=O\left((\log n)^{2}\right)$. Even though it is expected that $g(n)=O\left((\log n)^{1+\varepsilon}\right)$ for any $\varepsilon>0$, it seems that to prove $g(n)=O\left((\log n)^{\alpha}\right)$ with some $\alpha<2$ would be very difficult. Erdős, Gruber and Hammer [4] asked for a replacement of $S_{n}$ in Theorem $2^{*}$ by a set $S_{n}^{\prime}$ which would satisfy $\left|S_{n}^{\prime}\right|=O(\log n)$ as is expected from Theorem $1^{*}$. In connection with this problem, even if the expected order of $g(n)$ is established, Abbott's explicit construction falls short of our target.

In [2], Adhikari and Balasubramanian could give explicit construction of a set $S_{n}^{\prime} \subset \Delta_{n}^{2}$ from which $\Delta_{n}^{2}$ is visible, where $S_{n}^{\prime}$ satisfies

$$
\left|S_{n}^{\prime}\right|=O\left(\frac{\log n \cdot \log \log \log n}{\log \log n}\right)
$$

One observes that the order of $\left|S_{n}^{\prime}\right|$ not only satisfies (1), but also it improves on it by improving the upper bound of $f_{2}(n)$ thereof.

In a conference in RIMS, Kyoto, several mathematicians asked the first author about the answer to the similar question in higher dimensions.

In the present paper, we prove the following

THeOREM 3. One can give an explicit description of a set $X_{n} \subset \Delta_{n}^{3}$ from which $\Delta_{n}^{3}$ is visible, where $X_{n}$ satisfies

$$
\left|X_{n}\right|=O\left(\frac{\log n}{\log \log n}\right) .
$$

It is easy to see that the proof for the lower bound $\frac{\log n}{2 \log \log n}$ for $f_{2}(n)$ in [1] goes through in higher dimensions to yield the same lower bound for $f_{d}(n)$ for $d \geq 3$. Again, as will be clear from the proof of Theorem 3 (see Remark 1 after the proof of the theorem), for dimensions $d>3$ by trivial modifications of our proof one obtains the same result as in Theorem 3. Thus, for $d \geq 3$, the order problem for $f_{d}(n)$ is solved up to a constant factor. For $d=2$, it remains an open question whether the order of $f_{2}(n)$ obtained in [2] can be improved or not. 
Notations. For real $x$ we write $[x]$ for the integral part of $x$. We also use the notations $l_{i}(x), i \geq 1$, defined as follows:

$$
l_{1}(x)=\log x \quad \text { and } \quad l_{i}(x)=\log \left(l_{i-1}(x)\right) \quad \text { for } i \geq 2 .
$$

2. Proof of Theorem 3. Let $n$ be large and

$$
s=\left[D \sqrt{\frac{l_{1}(n)}{l_{2}(n)}}\right],
$$

where $D$ is a positive number such that $\sum_{p} 1 / p^{2}+2 / D^{2}<1$.

We take $X_{n}$ to be the set $\{(a, b, 1): 1 \leq a, b \leq s\} \cup\{(2,2,2)\}$. Given any $(x, y, z) \in \Delta_{n}^{3}$, we show that $(x, y, z)$ is visible from some point in $X_{n}$.

First we observe that given any $(x, y, z) \in \Delta_{n}^{3}$, if $z=1$, then $(x, y, z)$ is visible from $(2,2,2)$.

Now, we assume that $z \neq 1$. Then

$$
\begin{aligned}
\sum_{a, b=1}^{s} \sum_{((x-a),(y-b),(z-1))>1} 1 & \leq \sum_{a, b=1}^{s} \sum_{\substack{p \text { prime } \\
p \mid((x-a),(y-b),(z-1))}} 1 \\
& =\sum_{p \mid(z-1)} \sum_{\substack{1 \leq a, b \leq s \\
p|(x-a), p|(y-b)}} 1 \leq \sum_{p \mid(z-1)}\left(\frac{s}{p}+1\right)^{2} \\
& =s^{2} \sum_{p \mid(z-1)} \frac{1}{p^{2}}+2 s \sum_{p \mid(z-1)} \frac{1}{p}+\sum_{p \mid(z-1)} 1 \\
& <s^{2} \sum_{p} \frac{1}{p^{2}}+2 s \sum_{p<n} \frac{1}{p}+2 \frac{l_{1}(n)}{l_{2}(n)} \\
& <s^{2} \sum_{p} \frac{1}{p^{2}}+4 s l_{2}(n)+2 \frac{l_{1}(n)}{l_{2}(n)} \\
& =\left(\sum_{p} \frac{1}{p^{2}}+\frac{2}{D^{2}}\right) s^{2}+4 s l_{2}(n)<s^{2}
\end{aligned}
$$

for all sufficiently large $n$.

Therefore, there exist $a, b$ with $1 \leq a, b \leq s$ such that $((x-a)$, $(y-b),(z-1))=1$, which further implies that $(x, y, z)$ is visible from $(a, b, 1)$.

Hence, $\Delta_{n}^{3}$ is visible from $X_{n}$ as claimed and we get our theorem.

REMARK 1. It is clear from our proof that for $d>3, X_{n}$ could be replaced by $X_{n}^{d}=\left\{\left(a_{1}, \ldots, a_{d-1}, 1\right): 1 \leq a_{i} \leq s\right\} \cup\{(2,2, \ldots, 2)\}$ where $s=\left[D l_{1}(n) / l_{2}(n)\right]^{1 /(d-1)}$ with a suitable $D$. 
Remark 2. The $O$-constant in Theorem 3, which is about $D^{2}$, can be clearly brought down by handling the constants a little more carefully. Similarly, a glance at Abbott's proof for the lower bound in Theorem $1^{*}$ makes it clear that there, too, we can have a better constant than $1 / 2$. However here we are not much interested in those constants.

Acknowledgements. The work was done when the authors were visiting the Mathematical Institute of the Hungarian Academy of Sciences, Budapest. They are thankful to that institute and Professor Imre Z. Ruzsa for hospitality. The authors also acknowledge the supports by Hungarian National Science Foundation Grant No. 17433 (first author) and Fok Ying Tung Education Foundation and the National Natural Science Foundation of China (second author).

\section{References}

[1] H. L. Abbott, Some results in Combinatorial Geometry, Discrete Math. 9 (1974), 199-204.

[2] S. D. Adhikari and R. Balasubramanian, On a question regarding visibility of lattice points, Mathematika 43 (1996), 155-158.

[3] P. Erdős, On the integers relatively prime to $n$ and on a number-theoretic function considered by Jacobsthal, Math. Scand. 10 (1962), 163-170.

[4] P. Erdős, P. M. Gruber and J. Hammer, Lattice Points, Pitman Monographs Surveys Pure Appl. Math. 39, Wiley, New York, 1989.

[5] H. Iwaniec, On the error term in the linear sieve, Acta Arith. 19 (1971), 1-30.

[6] - On the problem of Jacobsthal, Demonstratio Math. 11 (1978), 225-231.

[7] H. J. Kanold, Über eine zahlentheoretische Funktion von Jacobsthal, Math. Ann. 170 (1967), 314-326.

[8] H. Stevens, On Jacobsthal's g(n)-function, ibid. 226 (1977), 95-97.

[9] R. C. Vaughan, On the order of magnitude of Jacobsthal's function, Proc. Edinburgh Math. Soc. 20 (1976-77), 329-331.

Mehta Research Institute

of Mathematics and Mathematical Physics

Chhatnag Road, Jhusi

Allahabad 211 019, India

E-mail: adhikari@mri.ernet.in
Department of Mathematics Nanjing Normal University Nanjing 210097, China E-mail: ygchen@pine.njnu.edu.cn 